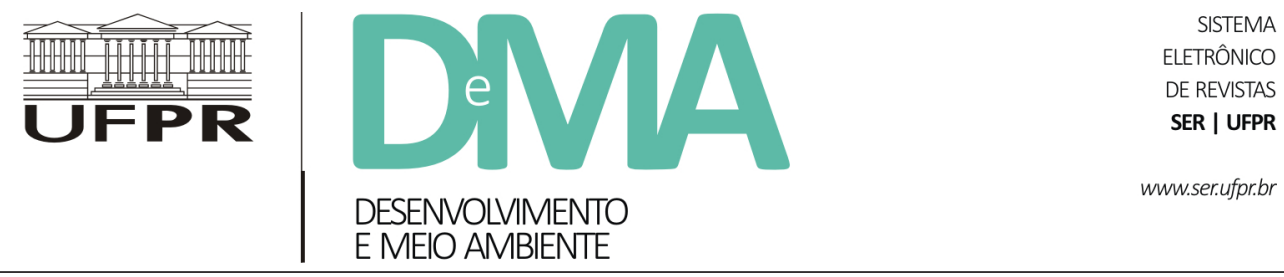

\title{
O processo participativo na Avaliação Ambiental Estratégica (AAE): aplicação de critérios de participação em relatórios de AAE da Bolívia
}

\section{The participation process of Strategic Environmental Assessment (SEA): application of participation criteria in Bolivia's SEA reports}

\author{
Aline Doria SANTI ${ }^{*}$, Caroline PICHARILLO ${ }^{1}$, Marcio Henrique BERTAZI ${ }^{1}$, Adriana Hofmann TREVISAN ${ }^{1}$ \\ ${ }^{1}$ Escola de Engenharia de São Carlos, Universidade de São Paulo (USP), São Carlos, SP, Brasil. \\ *E-mail de contato: alinedesanti@usp.br
}

Artigo recebido em 10 de maio de 2017, versão final aceita em 27 de fevereiro de 2018.

RESUMO: A Avaliação Ambiental Estratégica (AAE) é uma ferramenta proativa, sistemática e participativa que busca incorporar os componentes ambientais nos diferentes níveis estratégicos de decisão (Políticas, Planos e Programas), objetivando o desenvolvimento sustentável. Neste sentido, a participação pública possui um papel fundamental no que tange o fortalecimento das relações democráticas e de governança. Desse modo, diversas organizações internacionais estabeleceram critérios e princípios de participação pública em processos decisórios, os quais têm sido incorporados nas legislações de diversos países ao redor do mundo. O objetivo do presente trabalho consistiu em averiguar como o processo de participação pública é disposto nos relatórios de AAE da Bolívia, mediante a aplicação de critérios de participação estabelecidos no âmbito das organizações internacionais. O trabalho desenvolveu-se em três etapas de execução: Seleção de critérios da participação pública; Seleção dos relatórios de AAE da Bolívia; e Avaliação da participação pública nos relatórios selecionados. Ao todo, foram selecionados onze critérios de participação pública, os quais foram avaliados seguindo um sistema de notas estabelecido. De maneira geral, os quatro relatórios avaliados, no que concerne à inserção da participação pública, não possuem um caráter homogêneo - há relatórios bem descritos e organizados que atenderam satisfatoriamente os critérios propostos e relatórios que trouxeram informações superficiais. A escassez de vontade política, de informação disponível à população afetada e a debilidade do quadro legislativo, são fatores que limitam o envolvimento do público no processo decisório.

Palavras-chave: participação pública; AAE; Bolívia. 
ABSTRACT: Strategic Environmental Assessment (SEA) is a proactive, systematic and participatory tool that seeks to take into consideration the environmental components into different levels of strategic decisions (Policy, Plan and Programme making), aiming at a sustainable development. Therefore, public participation has a key role regarding the strengthening of democratic and governance relations. To this end, several international organizations established some criteria and principles about public participation in the SEA, which have been integrated in the legislation of several countries around the world. Thus, the present study aims to investigate how public participation has been incorporated in the SEA of Bolivia, through the application of participation criteria established by international organizations. The methodology followed three steps: 1) criteria selection of public participation; 2) selection of Bolivia's SEA reports; and 3) assessment of public participation in the reports selected. Eleven participation criteria were selected and assessed following an established grading system. Regarding public participation incorporation, the four assessed reports of Bolivia's SEA do not display a homogeneous nature: there are well described and organized reports that satisfactorily met the criteria, as well as others which displayed information that is more superficial. The results demonstrate that the lack of policy willingness, accessible information for the people interested and the weakness of the legislative framework can be considered the main limiting factors about public participation in the decision process.

Keywords: public participation; SEA; Bolivia.

\section{Introdução}

A Avaliação Ambiental Estratégica (AAE) é uma ferramenta proativa e sistemática que busca incorporar os componentes ambientais nos diferentes níveis estratégicos de decisão (Políticas, Planos e Programas - PPPs), permitindo à população afetada manifestar seus interesses e preocupações ainda na etapa de planejamento (Fischer, 2007). Este nível de participação busca fortalecer a governança, dar voz às comunidades locais, consolidar os preceitos democráticos e promover a aprendizagem mútua entre as partes interessadas, atuando desde o âmbito local para além das fronteiras nacionais (Kravchenko, 2002; Eversole, 2003; André et al., 2006; Ebesson, 2011). Desse modo, a AAE tem o compromisso latente de expandir o foco do tomador de decisão, a fim de incluir questões que ultrapassem seus interesses individuais, tornando-se um instrumento de gestão propício ao desenvolvimento sustentável (Therivel, 2004).
André et al. (2006) consideram três graus distintos de participação pública na AAE: no primeiro grau tem-se a Informação, na qual a participação é reconhecida como unilateral, possibilitando ao público ter acesso a informações relevantes sobre a AAE através dos meios de comunicação (e.g. rádio, jornal, televisão), porém isentos de expor suas sugestões. Em segunda instância, o grau da Consulta corresponde à abordagem participativa bilateral, na qual o público tem acesso às informações relevantes sobre o processo de AAE, permitindo-lhes apresentar suas opiniões e sugestões. Por fim, o grau mais elevado - de Co-decisão - reconhece a população como um parceiro atuante na tomada de decisão.

A inclusão da participação pública no processo decisório tem sido discutida e incorporada por diversas organizações internacionais, que buscam estabelecer critérios e princípios para seu desenvolvimento e consolidação. Dentre estas, destacam-se a United Nations Economic Commision for Europe (UNECE), a International Association for Impact Assessment (IAIA), a International Association for Public Participation (IAP2) (Berti \& Salvador, 
2014) e a Diretiva Europeia 2001/42/EC (EC, 2001).

Ao tratar especificamente sobre a participação pública nas questões ambientais, é importante citar - também - a Convention on Access to information, public participation in decision-making and Access to justice in environmental matters (Convenção de Aarthus); a qual procura garantir aos cidadãos direito à informação e à justiça ambiental, conduzindo ao progresso na proteção do meio ambiente e na melhoria da qualidade de vida (Mazzuoli \& Ayala, 2012).

A inserção das questões ambientais no processo de participação pública foi expressa, pela primeira vez, no princípio 10 da Declaração do Rio sobre Meio Ambiente e Desenvolvimento, em 1992. Além disso, o processo participativo foi reforçado no capítulo 23 da Agenda 21, estabelecendo que todo o indivíduo deve ter acesso adequado às informações relativas ao meio ambiente, bem como a oportunidade de participar dos processos decisórios. Desde então, as discussões e a implementação da participação pública têm se desenvolvido ao redor do mundo em função dos crescentes problemas ambientais (Mauerhofer, 2016; Ogihara et al., 2016).

O processo participativo vem sendo abordado em muitos relatórios de AAE nos países desenvolvidos e em desenvolvimento. Contudo, deve ser dada especial ênfase aos países em desenvolvimento, uma vez que estes passam por um processo lento de capacitação e institucionalização da AAE (Dalal-Clayton \& Sadler, 2005). Além disso, nestas áreas estão concentradas a maior parte das atividades econômicas primárias destinadas, inclusive, no abastecimento do mercado externo, e as áreas nativas remanescentes - vulneráveis à degradação (Pellin et al., 2011).
É importante destacar, também, que o gerenciamento de conflitos de interesses entre as partes envolvidas (Rega \& Baldizzone, 2015), bem como a atuação das agências multilaterais de desenvolvimento (AMD) - essas sendo, atualmente, as principais forças de capacitação e experiências práticas de AAE nos países em desenvolvimento -, resultam no enfraquecimento da participação pública na tomada de decisão. Pellin et al. (2011) constataram que a atuação das AMD, no âmbito da AAE, ocorre de forma homogênea entre os países mutuários quanto ao processo de concessão de financiamento, desconhecendo e desconsiderando o contexto social em que os mesmos se encontram inseridos. Infere-se que fatores políticos e econômicos se sobressaem aos fatores de interesse social, sendo as AAEs executadas - na maioria das vezes - em ocasião posterior ao planejamento do projeto, ou seja, raramente englobando as discussões estratégicas inerentes às fases de planos e programas, o que dificulta - ou, ainda, impede - a participação da população (Pellin et al., 2011).

Nesta perspectiva, considerar a participação pública - principalmente das populações mais afetadas - no âmbito da AAE, significa, portanto, reduzir os riscos às falhas no processo decisório, fortalecer e mobilizar a sociedade civil no apoio à implementação de políticas e planos, aumentar a confiança, melhorar a eficiência do planejamento governamental e conduzir a uma gestão e um manejo do meio ambiente de forma sustentável e consistente (Dalal-Clayton \& Sadler, 2005). Dessa forma, o presente artigo buscou averiguar como o processo de participação pública é disposto em alguns relatórios de AAE da Bolívia, mediante a aplicação de critérios de participação estabelecidos no âmbito das organizações internacionais: UNE- 
CE; IAIA; Banco Mundial (World Bank, 1999); IAP2 (IAP2, 2016); e Diretiva Europeia 2001/42/ EC (EC, 2001).

\subsection{A participação pública na AAE na} Bolivia

Desde 2004, a AAE na Bolívia vem se consolidando como "um instrumento de regulação e orientação para a tomada de decisões na planificação regional e nacional do desenvolvimento, orientado para a conservação da natureza" (Laats et al., 2013, p. 17). A partir de 2005, verificou-se um esforço por parte do Ministério do Meio Ambiente e das Águas, sob a égide da Netherlands Commission for Environmental Assessment (NCEA), de serem propostas medidas para a regulamentação da AAE no país - sendo que, em 2007, a AAE foi reconhecida pelo governo boliviano como política de prioridade nacional, de acordo com o Plano Plurianual de 2007-2011.

Em relação ao processo de participação pública no país, destaca-se o disposto pela Lei 1.551, de 20 de abril de 1994 (Bolívia, 1994), sancionada pelo Congresso Nacional Boliviano, a partir da qual se reconhece, promove e consolida a participação das comunidades indígenas, rurais e urbanas no processo de tomada de decisão, tanto por vias jurídicas e políticas quanto por vias econômicas. Esta regulamentação almeja a melhoria da qualidade de vida da população boliviana, a melhora na gestão dos recursos públicos e o fortalecimento dos instrumentos políticos e econômicos necessários para aperfeiçoar a democracia representativa.

A questão da participação pública na $\mathrm{AAE}$, por sua vez, é inserida no Art. 13 do Projeto de
Regularização da Avaliação Ambiental Estratégica, a partir do qual se reconhece a AAE como um instrumento de planificação integral estratégico, anterior e posteriormente ao processo de construção ou implementação de PPPs, considerando todos os níveis estratégicos de planejamento do Estado (Ncea, 2013). O respectivo artigo garante o exercício do direito à participação pública no processo de elaboração da AAE, sendo previstos um Plano de Participação Pública e Gestão de Conflitos e um Plano de Comunicação que permitam o fluxo de informações transparentes, participativas e não discriminatórias. Tal procedimento vale-se das dívidas históricas no processo socioeconômico e ambiental do país (Bethell, 1997), reafirmando o direito à participação dos povos indígenas e campesinos, assegurando, dessa forma, seus valores, cultura e formas de organização social (Ncea, 2013).

É importante salientar que a existência deste documento legal no país, o qual discorre sobre a participação pública no processo de tomada de decisão, é fundamental para estimular e reforçar a atuação da população junto à elaboração da AAE. Porém, a inexistência de uma legislação que trate exclusivamente do processo de $\mathrm{AAE}$ no âmbito boliviano pode gerar insuficiências e omissões com relação ao processo participativo. Além disso, o Ministério de Meio Ambiente e Água da Bolívia tem, ainda, identificado uma fragilidade quanto à incorporação das conclusões ou recomendações das AAEs que têm sido realizadas. Curiosamente, parece haver uma correspondência direta com a aplicação ainda incipiente de mecanismos de participação efetivos e acesso à informação de modo geral (Ncea, 2013). 


\section{Metodologia}

A metodologia adotada neste artigo baseou-se no trabalho de Berti, Dibo \& Sanches (2012), sendo estruturada em três etapas de execução: (I) Seleção e adaptação de critérios da participação pública; (II) Seleção dos relatórios de AAE da Bolívia; e (III) Avaliação da participação pública nos relatórios.

\subsection{Seleção de critérios de participação pública}

Esta etapa incluiu o levantamento de critérios e princípios de participação pública, mediante revisão de documentos e diretrizes pertencentes às seguintes organizações internacionais: UNECE; IAIA; Banco Mundial (World Bank); IAP2; e Diretiva Europeia 2001/42/EC.

\subsection{Seleção dos relatórios de AAE da Bolívia}

Os relatórios de AAE da Bolívia foram selecionados de acordo com os seguintes critérios, estabelecidos por Berti \& Salvador (2014), a saber: (I) Relatórios de AAE disponíveis na internet para acesso público; (II)
Relatórios de AAE finalizados; e (III) Relatórios de AAE completos ${ }^{1}$.

\subsection{Avaliação da participação pública nos relatórios}

Nesta etapa, os critérios de participação pública, selecionados e adaptados na primeira etapa, foram aplicados na avaliação dos relatórios de AAE da Bolívia selecionados. É importante ressaltar que, embora o relatório como um todo tenha embasado as discussões dos resultados, a avaliação dos relatórios foi direcionada às seções e subseções dedicadas exclusivamente ao processo de participação pública na $\mathrm{AAE}$, sendo o método de avaliação uma análise exploratória das informações presentes nos relatórios - sem averiguações em campo dos desdobramentos e sem considerações efetivas do processo participativo.

Os relatórios de $\mathrm{AAE}$ da Bolívia foram avaliados de acordo com a escala de notas (de A a G), proposta por Fischer (2010), sendo atribuído uma nota a cada um dos critérios de participação pública, como descrito na Tabela 1.

TABELA 1 - Notas para a avaliação da participação pública nos relatórios de AAE.

\begin{tabular}{cl}
\hline Nota & \multicolumn{1}{c}{ Informações sobre o Relatório } \\
\hline A & Apresentou bom desempenho, sem omissões relevantes. \\
B & Foi satisfatório e completo, com pequenas omissões e/ou inadequações. \\
C & Foi satisfatório, apesar de algumas omissões ou inadequações. \\
D & $\begin{array}{l}\text { Indicou que partes foram atendidas, mas, no geral, foi insatisfatório devido às omissões e/ou inadequações/insuficiên- } \\
\text { cia de dados. } \\
\text { E }\end{array} \quad$ Não foi satisfatório, com omissões significativas e/ou inadequações de dados. \\
F & Foi muito insatisfatório, com importantes questões fracamente atendidas. \\
G & Não atendeu a critério algum.
\end{tabular}

FONTE: Fischer (2010).

${ }^{1}$ Relatórios que descrevam todas as fases do processo de AAE. 


\section{Resultados e discussão}

$\mathrm{Na}$ análise dos documentos das organizações descritas previamente, foram identificados, ao todo, onze critérios distintos sobre participação pública na AAE, os quais estão apresentados na Tabela 2. $\mathrm{O}$ décimo primeiro critério foi estruturado pelos autores após verificarem que a literatura apontava com relativa frequência que o processo de participação pública consistia em uma série de diálogos que, tradicionalmente, não ocorriam.

Por esta razão, era esperado que tais dificuldades aparecessem na elaboração dos relatórios, prevendo-se que a sua menção evidencie como os processos - que são vistos como não construtivos - podem reduzir o apoio e a legitimidade das pro-

TABELA 2 - Critérios selecionados para avaliação da participação pública em relatórios de AAE.

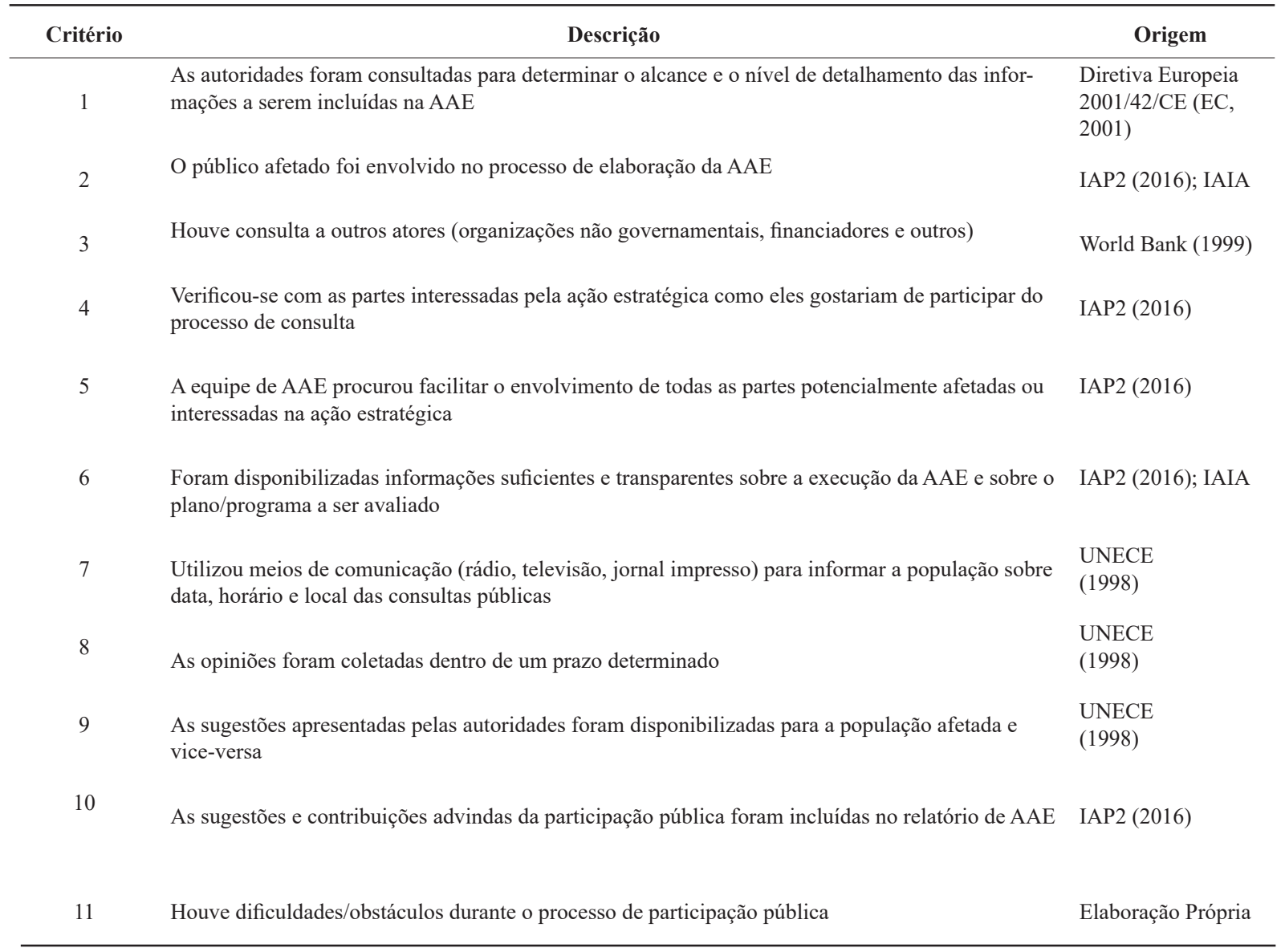

FONTE: Elaboração própria. 
postas (Tuler \& Webler, 1999). A ausência dessas dificuldades nos relatórios pode indicar que não estejam cumprindo exatamente as prerrogativas esperadas para a participação pública, apenas enviesando-se em um cumprimento de etapas, no qual a atividade do cidadão e da cidadã resume-se a ter "participado de uma participação" (Arnstein, 1969).
A avaliação dos relatórios de $\mathrm{AAE}$, que se enquadraram nos parâmetros pré-estabelecidos por Berti \& Salvador (2014) (Tabela 3), demonstrou que há diferenças significativas entre os relatórios no que diz respeito ao atendimento dos critérios de participação pública analisados, conforme observado na Figura 1.

TABELA 3 - Relatórios de AAE selecionados.

\begin{tabular}{lcc}
\hline \multicolumn{1}{c}{ Relatório de AAE } & Ano & Disponibilidade \\
\hline Avaliação Ambiental Estratégica do Corredor Santa Cruz Puerto Suarez (CSCPS) & 2002 & $\underline{\text { https://goo.gl/XISbAw }}$ \\
Avaliação Ambiental Estratégica do Corredor Norte (CN) & 2006 & $\underline{\text { https://goo.gl/TPDR3k }}$ \\
Avaliação Ambiental Estratégica do Programa Florestal Baba Carapa (PFBC) & 2011 & $\underline{\text { http://goo.gl/YoZKm7 }}$ \\
$\begin{array}{l}\text { Avaliação Ambiental Estratégica para o desenvolvimento integral sustentável do } \\
\text { território indígena Parque Nacional Isiboro Sécure (TIPNIS) }\end{array}$ & 2011 & $\underline{\text { http://goo.gl/DUPZ77 }}$ \\
\hline
\end{tabular}

FONTE: Elaboração própria.

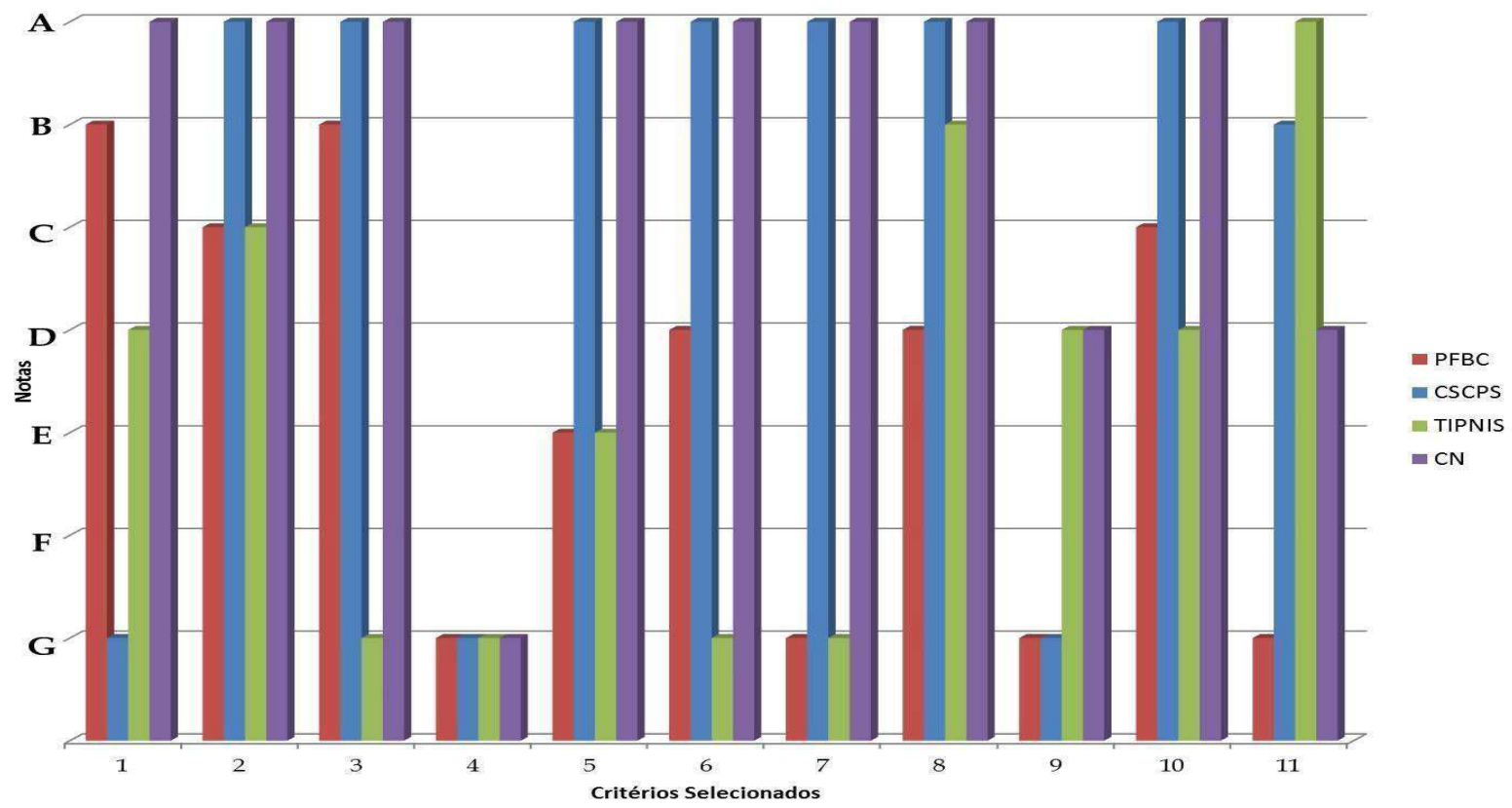

FIGURA 1 - Resultado da avaliação dos relatórios de AAE com base nos critérios de participação pública selecionados. FONTE: Elaboração própria. 
De acordo com o disposto na Figura 1, verifica-se que o critério 4 foi desfavorável para todos os casos avaliados, ou seja, em nenhum dos relatórios avaliados consta o modo e as circunstâncias em que as partes interessadas gostariam de participar do processo de elaboração da AAE. Tal estratégia é fundamental para que o canal de participação a ser utilizado atenda as demandas e as aspirações do público, de forma a estimular e otimizar a atuação das comunidades no processo decisório (Gauthier et al., 2011).

Walker et al. (2014), por exemplo, em seu trabalho realizado em uma comunidade local no Quênia, detectaram um desconforto por parte de membros dessa comunidade que, ao participarem de reuniões de elaboração de uma AAE, se sentiram coagidos ao expressar suas opiniões frente às autoridades locais ali presentes. Infere-se, pois, que os métodos e técnicas escolhidos para a participação pública devem levar em consideração o contexto político, social e cultural das populações envolvidas, tendo como objetivo obter o maior número de participações possíveis, bem como evitar vieses e informações errôneas.

A participação pública no processo de elaboração da $\mathrm{AAE}$ pode ocorrer por meio de diversos mecanismos, a saber: reuniões, entrevistas, seminários, oficinas, audiências públicas, consultas públicas, questionários, entre outros. Neste sentido, é imprescindível que os métodos e técnicas utilizados encorajem o diálogo e a reflexão crítica, permitindo aos participantes expressarem adequadamente seus pontos de vista, de forma acessível e compreensível para todos - independentemente do grau de instrução, cultura, idioma, etc. (Walker et al., 2014). Além disso, a execução de duas ou mais "rodadas de consulta pública" é necessária, de modo que haja troca de experiências e informações entre as partes envolvidas, considerando assim diferentes pontos de vista, além de preservar a transparência das informações coletadas (Devuyst et al., 2000).

No processo de participação pública do $\mathrm{CN}$, foram realizadas três rodadas de consulta pública bem detalhadas no relatório. A primeira rodada foi planejada para obter informações importantes ao diagnóstico socioeconômico e ambiental de cada região ao longo do trajeto do corredor norte. Por outro lado, a segunda rodada objetivou apresentar os resultados do diagnóstico, assim como as diretrizes geradas para compor as alternativas do plano em questão. A terceira rodada, por sua vez, correspondeu à apresentação dos resultados principais da AAE, a partir da qual o plano foi ratificado.

Tal mecanismo também foi observado no relatório de AAE do Corredor Santa Cruz Puerto Suarez (CSCPS), o qual realizou duas "rodadas" de consulta pública (i.e. a primeira relacionada à apresentação do plano e à coleta de informações para o diagnóstico socioeconômico e ambiental da região; e a segunda de caráter consultivo), envolvendo mais de mil participantes. O que se observa, portanto, são procedimentos de debate e inclusão das sugestões do público desde o início do processo de elaboração da AAE, tornando-a satisfatória (Walker et al., 2014). Isso, de modo geral, influenciou no bom desempenho dos relatórios do $\mathrm{CN}$ e do CSCPS. A participação prévia e contínua é determinante para que a política pública seja considerada mais efetiva, além de se prevenir ou mitigar conflitos e consequências ambientais adversas (Coletti, 2012).

Além das rodadas de consultas públicas, foram realizados - no caso da AAE do $\mathrm{CN}$ - encontros regionais (incluindo a sociedade civil e as comunidades locais), reuniões setoriais (produtores, 
empresas privadas) e reuniões com representantes de órgãos governamentais. Já no relatório de AAE do CSCPS, foram descritas oficinas, seminários e reuniões técnicas com organizações indígenas, líderes locais, autoridades, produtores, camponeses e representantes da sociedade civil.

No tocante aos outros relatórios avaliados, a AAE do Programa Florestal Baba Carapa (PFBC) realizou oficinas, reuniões e entrevistas que parecem ter considerado a opinião dos atores, sobretudo da alçada governamental. Assim, influenciando - principalmente - na incorporação de temáticas vinculadas à sustentabilidade, o que explica que cerca de $18 \%$ da avaliação tenha sido "B" (critérios 01 e 03 ). Entretanto, menciona-se apenas o número de oficinas realizadas (cinco), sem o detalhamento de como foram realizadas as reuniões e entrevistas. Há, também, dificuldades latentes com relação à compreensão entre o que foi sugerido e o que foi incorporado no relatório (critério 10).

Em relação à $\mathrm{AAE}$ do Parque Nacional Isiboro Sécure (TIPNIS), as principais conclusões e recomendações da AAE se deram através dos estudos, reflexões coletivas realizadas em 33 oficinas comunitárias e a partir de inúmeras reuniões entre o consultor e o serviço nacional de áreas protegidas - SERNAP. No entanto, o relatório somente cita que essas informações foram inseridas na AAE, não especificando de modo transparente como foram incorporadas e quais foram as possíveis contribuições ao plano em questão.

Para o critério 09, que trata da "troca de informações" entre a população afetada e os representantes de órgãos governamentais, o conteúdo do relatório do $\mathrm{CN}$, por exemplo, descreve que as reuniões e oficinas realizadas com a população local foram todas registradas e, posteriormente, disponi- bilizadas na internet. Porém, não foi evidenciado se tais informações foram tratadas pelas autoridades, assim como não fica claro se as sugestões levantadas pela comunidade local foram dispostas para o público em geral.

A pesquisadora Maria da Glória Gohn (2011) aponta que a questão da institucionalidade das práticas que ocorrem no âmbito civil nos espaços públicos tem sido um processo sociopolítico de relevância em vários países da América Latina, sobretudo a partir do final dos anos noventa. Boa parte da interação política decorrente deste quadro tem se dado via políticas institucionalizadas. Nos países da América Latina em que essa prática tem ocorrido - e a Bolívia está inserida - o elemento catalisador é a questão da territorialização e da espacialização, além da inserção dos conflitos que se dão nas fronteiras da política, da sociedade e da cultura.

Frente à descrição dos mecanismos de participação, utilizados em cada processo de AAE em análise, destaca-se que a inclusão de todas as partes afetadas e interessadas no processo (e.g. representantes das comunidades locais, governo, instituições privadas, grupos setoriais, organizações não governamentais, entre outros), tal como observado anteriormente, reforça o potencial de ação da AAE principalmente quando alcança grupos normalmente suprimidos das decisões políticas e econômicas de um país (e.g. povos indígenas, mulheres e jovens) (Arnstein, 1969; Rega \& Bonifazi, 2013).

Na América Latina, o território tem se tornado referência central "para os projetos de agregação das ações coletivas e não mais as ideologias ou correntes de opiniões políticas" (Gohn, 2011, p. 226). Isso se dá porque o território tem se comportado como uma espécie de aglutinador das demandas da ancestralidade dos povos, seus idiomas, culturas e 
símbolos. A inclusão da população marginalizada aumenta seu engajamento sobre questões ambientais, além de fortalecer indivíduos e comunidades na busca por seus direitos. Trata-se, pois, de um aspecto forte do processo de participação pública (Walker et al., 2014) e tal proposição foi observada no relatório de AAE do PFBC, CN e CSPS, que envolveu representantes das comunidades locais, de instituições governamentais e organizações não governamentais, povos indígenas e camponeses.

No relatório de TIPNIS é salientada a importância da inclusão da população indígena no processo de elaboração da $\mathrm{AAE}$, de modo a potencializar a participação dessas comunidades em ações estratégicas de desenvolvimento territorial e socioeconômico. Entretanto, o relatório não apresenta informações consistentes referentes à participação desta comunidade. Assim, caracteriza-se como um relatório fraco diante do processo de inclusão da população marginalizada (Walker $e t$ al., 2014). Devlin \& Yap (2008) acrescentam, ainda, que a participação pública na AAE pode contribuir efetivamente com a redução de conflitos sociais, garantindo a representação de diferentes interesses, o que não foi identificado na AAE do TIPNIS.

Não obstante, o relatório de TIPNIS foi o único que apresentou nota "A" para o critério 11 , visto que foram relatadas as dificuldades encontradas durante a etapa de coleta de informações com as partes envolvidas. Já no relatório do $\mathrm{CN}$, não são especificadas dificuldades intrínsecas ao processo de participação pública, relatando-se apenas a ocorrência de problemas internos na Bolívia, no momento da elaboração da AAE, os quais interferiram apenas no período de execução das "rodadas de consulta pública". Torna-se relevante evidenciar, ainda, que o relatório do $\mathrm{PFBC}$ não menciona - ou ao menos não são evidentes - as dificuldades ou os obstáculos durante o processo de consulta pública.

Outro ponto importante para ser observado no processo de participação pública, diz respeito ao estabelecimento de um prazo razoável por parte dos proponentes da ação, para que as partes envolvidas tenham tempo suficiente para elaborar seus comentários e sugestões. Exprime-se, assim, igual necessidade de se disponibilizar, com antecedência, informações sobre a AAE e os PPPs, que sejam transparentes e em linguagem compreensível a todos os interessados (Berti et al., 2012; Walker et al., 2014). Tais proposições são expressas nos critérios 06 e 08 e foram evidenciadas claramente nos relatórios do $\mathrm{CN}$ e CSPS.

No que concerne à divulgação de informações relacionadas ao processo participativo, a UNECE (1998) orienta que os meios de comunicação (e.g. rádio, televisão, jornais impressos) devem ser amplamente utilizados para este fim, além de divulgar data, horário e local das atividades da AAE - estimulando a população a participar do processo.

Dentre os relatórios de AAE avaliados, o $\mathrm{CN}$ e CSCPS apresentam informações sobre a utilização desses meios para divulgar as rodadas de consulta pública e, no caso do CSCPS, além dos meios de comunicação anteriormente citados, a população foi mobilizada mediante visitas dos membros da equipe de AAE - diferentemente do observado nos relatórios de TIPNIS e PFBC. As convocações para as audiências públicas, por exemplo, devem levar em consideração as exigências de publicidade e de acessibilidade da população, visto que convocações encontradas somente em meios oficiais do Estado, ou o estabelecimento das audiências em horários difíceis para a participação dos grupos sociais atingidos (e.g. período noturno), mascaram a real 
efetividade da participação do público no processo de tomada de decisão (Coletti, 2012).

Com relação especificamente ao relatório de AAE do PFBC, verificou-se que a participação pública se deu de modo transversal e a metodologia aplicada deu-se pela perspectiva qualitativa, ainda que essas afirmações tenham sido feitas no plano teórico. O enfoque metodológico procurou enfatizar o reconhecimento do papel central da linguagem e do discurso, embora não tenha ficado evidente como esse reconhecimento tenha se dado na prática da participação e da consulta, tal como se verifica na baixa avaliação dos critérios 04,05 e 07 .

No contexto do PFBC, Tuler \& Webler (1999) sugeriram que um programa florestal deva optar por uma gestão construtiva, isto é, dar a garantia de que um caminho producente seja assegurado ao invés de todas as opções selecionadas durante a consulta tornarem-se bloqueadas por conflitos de interesse. Evidentemente, essa consulta deve permitir as melhores relações possíveis entre os grupos de interesse da região em questão, fato este não observado no respectivo relatório, no qual foram privilegiados apenas atores do setor governamental. A pouca integração entre as informações, bem como a incerteza com relação a aceitação ou não das informações colhidas no decorrer do processo de AAE - que culminou no relatório - e a seletividade dos atores consultados, justificam a avaliação mediana do relatório (com 63,6\% abaixo da nota "C").

Cabe ressaltar que a avaliação desfavorável de alguns critérios - seja ela na etapa de planejamento da participação pública, na disponibilização de informações sobre os mecanismos participativos ou até mesmo no relato das dificuldades enfrentadas -, que poderiam auxiliar na melhoria contínua do processo, comprometem em diferentes instâncias a suficiência da participação pública nos processos decisórios.

\section{Considerações finais}

De forma geral, é possível inferir que os relatórios de AAE da Bolívia avaliados, excetuando-se o relatório do TIPNIS, atenderam satisfatoriamente os critérios de participação propostos. Contudo, os resultados demonstram que estes não possuem um caráter homogêneo. O CN e do CSCP, por exemplo, apresentam informações detalhadas sobre a participação pública na AAE, o que diverge das assertivas feitas por Weiland (2010), Deasley et al. (2011) e Rega \& Baldizzone (2015), de que os níveis de participação pública na $\mathrm{AAE}$ são baixos e limitados; enquanto que as informações apresentadas nos relatórios de PFBC e TIPNIS são mais superficiais.

É importante ressaltar que a falta de vontade política, a ausência de informação disponível à população afetada e a debilidade do quadro legislativo, são fatores que limitam o envolvimento do público no processo de elaboração da AAE (Rega \& Baldizzone, 2015), estando estes fatos diretamente relacionados com as omissões identificadas no relatório do TIPNIS.

A Nova Constituição Política do Estado (NCPE), aprovada em 2010 na Bolívia, redefiniu o conceito de Estado, assim como o de cidadania, a partir de uma lógica plurinacional, multicultural e comunitária. Trata-se de uma democracia não apenas representativa, mas também participativa. Katya A. Rudón (2010) aponta que embora o NCPE reconheça o direito à informação, não garante o seu controle direto. 
Como articulado por Costa et al. (2009), a construção de políticas que atendam a demanda de todas as partes interessadas, associada com a criação de uma representatividade mais ativa, é um dos maiores desafios da participação pública, implicando em novos formatos de articulação entre os atores e o estado. Neste sentido, estruturas legais mais sólidas, requisitos elevados de participação e a melhor orientação técnica, são ações necessárias para melhorar o envolvimento do público em processos decisórios.

As formas de participação podem não determinar o sucesso do resultado (Chess \& Purcell, 1999), devendo estas estarem interligadas a todo o processo de construção da $\mathrm{AAE}$ para que se tornem efetivas (Arnstein, 1969). Tais assertivas cabem ao processo de elaboração da AAE na Bolívia, a qual demonstra discrepância entre a estrutura e semântica dos relatórios e, também, quanto ao envolvimento do público neste processo.

Por fim, é importante salientar que as análises conduzidas neste artigo foram exploratórias, baseadas nas informações disponíveis nos relatórios de AAE, sem verificação in loco que subsidiasse a análise da credibilidade do relatório, bem como da efetividade e implicações dos processos participativos praticados em cada caso. Dessa forma, análises mais detalhadas em nível local são necessárias, de modo a obter uma maior articulação das políticas públicas - almejando a descentralização de poder e, consequentemente, o fortalecimento da democracia participativa.

\section{Referências}

André, P.; Enserink, B.; Connor, D.; Croal, P. Public Participation International Best Practice Principles. International
Association for Impact Assessment. Special Publication Series, 4, 2006. Disponível em: <https://www.iaia.org/ uploads/pdf/SP4.pdf>

Arnstein, S. R. A ladder of citizen participation. Journal of the American Institute of Planners, 35(4), 216-224, 1969. doi: 10.1080/01944366908977225

Berti, M. J. F.; Dibo, A. P. A.; Sanches, R. Public Participation in the SEA Process of Brazil. In: 32nd Annual Meeting of the International Association for Impact Assessment. Energy Future 'The Role of Impact Assessment'. Porto: Portugal, 2012.

Berti, M. J. F.; Salvador, N. N. B. Análise do processo participativo na avaliação ambiental estratégica no Brasil. Revista Brasileira de Ciências Ambientais, 33, 73-84, 2014. Disponível em: < http://abes-dn.org.br/publicacoes/ rbciamb/PDFs/33-07.pdf $>$

Bethell, L. Historia de América Latina: política y sociedad desde 1930. Tradução de Jordi Beltran. Barcelona: Crítica, 1997.

Bolívia. Lei n. 1551, de 20 de Abril de 1994. Lei de Participação Popular. Câmara do Congresso Nacional, La Paz, 20 Abr. 1994.

Chess, C.; Purcell, K. Public participation and the environment: do we know what Works? Environmental Science \& Technology, 33(16), 2685-2692, 1999. doi: 10.1021/ es980500g

Coletti, R. N. A participação da sociedade civil em instrumentos da política ambiental brasileira. Desenvolvimento e Meio Ambiente, 25, 39-51, 2012. doi: 10.5380/dma. v25i0.25544

Costa, H. A.; Bursztyb, M. A.; Nascimento, E. P. Participação social em processos de Avaliação Ambiental Estratégica. Sociedade e Estado, 24(1), 89-113, 2009. doi: 10.1590/ S0102-69922009000100005

Dalal-Clayton, B.; Sadler, B. Strategic environmental assessment: a sourcebook and reference guide to international experience. London. Earthscan. 470 p. 2005.

Deasley, N.; Billett, S.; Watt, S. C. The Scottish strategic environmental assessment review. Stirling: Scottish Environment Protection Agency with Historic Scotland and 
Scottish Natural Heritage, 2011. Disponível em: $<$ https:// www.sepa.org.uk/media/27556/sea-review_summary.pdf $>$

Devlin, J. F.; Yap, N. T. Contentious politics in environmental assessment: blocked projects and winning coalitions. Impact Assessment Project Appraisal, 26(1), 17-27, 2008. doi: 10.3152/146155108X279939

Devuyst, D.; Wijngaarden, T. V. Hens, L. Implementation of SEA in Flanders:Attitudes of key stakeholders and a user-friendly methodology. Environmental Impact Assesment Review, 20, 65-83, 2000. doi: 10.1016/S0195-9255(99)00013-X

Ebesson, J. A modest contribution to environmental democracy and justice in transboundary contexts: the combined impact of the Espoo Convention and Aarhus Convention. RACIEL, 20(3), 248-257, 2011. doi: 10.1111/j. 1467-9388.2011.00728.x

EC - European Commission. Directive 2001/42/EC of the European Parliament and of the Council on the Assessment of the Effects of Certain Plans and Programmes on the Environment. Luxembourg, 21.7.2001. Disponível em: $<$ http://ec.europa.eu/environment/archives/eia/pdf/030923_ sea_guidance.pdf $>$

Eversole, R. Managing the pitfalls of participatory development: some insight from Australia. World Development, 31(5), 781-795, 2003. doi: 10.1016/S0305750X(03)00018-4

Fischer, T. B. The Theory and Practice of Strategic Environmental Assesment: Towards a More Systematic Approach. London. P. 209, 2007.

Fischer, T. B. Reviewing the quality of strategic environmental assessment reports for English spatial plan core strategies. Environmental Impact Assessment Review, 30, 62-69, 2010. doi: 10.1016/j.eiar.2009.04.002

Gauthier, M.; Simard, L.; Waaub, J. P. Public participation in strategic environmental assessment (SEA): critical review and the Quebec (Canada) approach. Environmental Impact Assessment Review, 31, 48-60, 2011. doi: 10.1016/j. eiar.2010.01.006

Gohn, M. G. Participação de representantes da sociedade civil na esfera pública na América Latina.
Politica \& Sociedade, 10(18), 233-244, 2011. doi: 10.5007/2175-7984.2011v10n18p233

IAP2 - International Association for Public Participation. IAP2 Core Values. 2016. Disponível em: <http://www.iap2. org/?page $=\mathrm{A} 4>$. Acesso em: jun. 2017.

Kravchenko, S. Effective public participation in the preparation of policies and legislation. Environmental Policy and Law, 32(5), 204-208, 2002.

Laats, H.; Hurtado, G. M.; Cabaleiro, P.; Dalal-Clayton, B.; Paredes, X.; Coello, J. La Experiencia de las evaluaciones ambientales estratégicas en Bolivia, p.. 115, 2013. Disponível em: <http://www.bankinformationcenter.org/ wp-content/uploads/2013/07/Experiencias-EAE-en-Bolivia.pdf>. Acesso em: mai. 2017.

Mauerhofer, V. Public participation in environmental matters: Compendium challenges and chances globally. Land Use Policy, 52, 481-491, 2016. doi: 10.1016/j.landusepol.2014.12.012

Mazzuoli, V. O.; Ayala, P. A. Cooperação Internacional para a preservação do meio ambiente: o direito brasileiro e a Convenção de Aarhus. Revista Direito GV, 8(1), 297-328, 2012. doi: 10.1590/S1808-24322012000100012

Ncea - Netherlands Commission for Environmental Assessment. Experiencia Boliviana en EAE, el marco legal y manuales, 2013. Disponível em: <http://www.eia.nl/en/ countries/sa/bolivia/sea>. Acesso em: jun. 2017.

Ogihara, A.; Shimaoka, M.; Roppongi, H. Potentialities for a regional public participation framework in Asia: An environmental assessment perspective. Land Use Policy, 52, 535-542, 2016. doi: 10.1016/j.landusepol.2015.09.029

Pellin, A.; Lemos, C. C.; Tachard, A.; Oliveira, I. S. D.; Souza, M. P. Avaliação Ambiental Estratégica no Brasil: considerações a respeito do papel das agências multilaterais de desenvolvimento. Engenharia Sanitária e Ambiental, 16(1), 27-36, 2011. doi: 10.1590/S1413-41522011000100006

Rega, C.; Baldizzone, G. Public participation in Strategic Environmental Assesment: A practitioners perspective. Environmental Impact Assessment Review, 50, 105-115, 2015. doi: 10.1016/j.eiar.2014.09.007

Rega, C.; Bonifazi, A. Strategic environmental as- 
sessment and spatial planning in Italy: sustainability, integration and democracy. Journal of Environmental Planning Management, 57(9), 1333-1358, 2013. doi: 10.1080/09640568.2013.804404

Rudón, K. A. Bolivia: pensar la libertad desde los medios y la democracia. Revista Latinoamericana de Comunicación Chasqui, 109, 34-38, 2010. Disponível em: < http://www.revistachasqui.org/index.php/chasqui/article/view/119/131>

Therivel, R. Strategic Environmental Assessment in Action. London: Earthscan, 2004.

Tuler, S.; Webler, T. Voices from the forest: what participants expect of a public participation process. Society \& Natural Resources: An International Journal, 15(5), 437453, 1999. doi: 10.1080/089419299279524

UNECE - United Nations Economic Commision for Europe. Convention on access to information, public participation in decision-making and access to justice in environmental matters. 1998. Disponível em: <http://www. unece.org/fileadmin/DAM/env/pp/documents/cep43e.pdf>. Acesso em: jun. 2017.
Walker, H.; Sinclair, A. J.; Spaling, H. Public participation in and learning through SEA in Kenya. Environmental Impact Assessment Review, 45, 1-9, 2014. doi: 10.1016/j. eiar.2013.10.003

Weiland, U. Strategic environmental assessment in Germany — practice and open questions. Environmental Impact Assessment Review, 30, 211-217, 2010. doi: 10.1016/j. eiar.2009.08.010

World Bank. Public Consultation in the EA Process: A Strategic Approach. Environmental Assessment Sourcebook, n.23, 1999. Disponível em:< http://siteresources.worldbank. org/INTSAFEPOL/1142947-1118039086869/20526299/ Update26PublicConsultationInTheEAProcessAStrategicApproachMay 1999.pdf> 